\title{
Eccentric-only versus concentric-only resistance training effects on biochemical and physiological parameters in patients with type 2 diabetes
}

\author{
Christine Kudiarasu ${ }^{1,2^{*}}$ (D, Wafina Rohadhia ${ }^{1}$, Yoshihiro Katsura ${ }^{3}$, Tomoko Koeda ${ }^{4}$, Favil Singh ${ }^{1,2}$ and \\ Kazunori Nosaka ${ }^{1,2}$
}

\begin{abstract}
Background: The benefits of resistance training for patients with type 2 diabetes (T2D) are well documented; however, the effects of exercise with different muscle contraction types such as eccentric versus concentric contractions on physiological outcomes for this population are not clear. This study compared eccentric-only (ECC) and concentric-only resistance training (CON) to test the hypothesis that ECC would be superior to CON to improve insulin sensitivity, lipid profile, body composition, muscle strength and physical function of patients with T2D.
\end{abstract}

Methods: Adults with T2D (50-79 years) were allocated to the ECC $(n=9)$ or CON group $(n=9)$. Resistance exercises (chest press, lateral pulldown, bicep curl, triceps extension, leg extension, leg curl, calf raise, abdominal crunch) consisting of $2-3$ sets of 10 eccentric-only ( 5 s) or concentric-only contractions (1-2 s) was performed twice a week for 12 weeks. Changes in blood biomarkers, body composition, muscle strength and physical function from pre- to post-intervention were compared between groups.

Results: Overall rating of perceived exertion (RPE, 1-10 Borg scale) was lower ( $p<0.05)$ for ECC $(2.9 \pm 1.2)$ than CON $(5.4 \pm 1.1)$. No significant changes in blood biomarkers were found for both groups. Lean mass increased [effect size $(E S)=0.148$, ECC $3.2 \pm 6.9 \% ; C O N ~ 3.6 \pm 2.3 \%]$, and fat mass decreased $(E S=0.545$, ECC $-6.1 \pm 12.4 \%$; $C O N$ $-7.1 \pm 16.4 \%)(p<0.05)$ similarly. One-repetition maximal strength of each exercise increased $(p<0.05)$ for both ECC $(12-37 \%)$ and $C O N(27-68 \%)$. Both groups improved $(p<0.05) 6$-min walk distance (ES $=0.083$, ECC 12.2 $\pm 2.3 \%$; CON $12.5 \pm 15.3 \%)$ and chair rise time $(E S=0.463, E C C-13.4 \pm 25.4 \%$; $C O N-20.0 \pm 53.3 \%)$ but only $E C C$ improved $(p<0.05)$ the timed up-and-go test $(-11.3 \pm 13.6 \%$, ES 0.014). No significant changes in balance tests were found for both groups.

Conclusion: These results did not fully support the hypothesis but showed that ECC was as effective as CON to improve body composition, muscle strength, and physical function with lesser RPE. Future studies should investigate whether larger differences between ECC and CON are evident when increasing the exercise frequency and matching the intensities of the two-exercise protocols.

Trial registration ACTRN12621001026819 (retrospectively registered on 5th Aug 2021).

*Correspondence: c.kudiarasu@ecu.edu.au

${ }^{2}$ Exercise Medicine Research Institute, Edith Cowan University, Joondalup, WA, Australia

Full list of author information is available at the end of the article permits use, sharing, adaptation, distribution and reproduction in any medium or format, as long as you give appropriate credit to the original author(s) and the source, provide a link to the Creative Commons licence, and indicate if changes were made. The images or other third party material in this article are included in the article's Creative Commons licence, unless indicated otherwise in a credit line to the material. If material is not included in the article's Creative Commons licence and your intended use is not permitted by statutory regulation or exceeds the permitted use, you will need to obtain permission directly from the copyright holder. To view a copy of this licence, visit http://creativecommons.org/licenses/by/4.0/. The Creative Commons Public Domain Dedication waiver (http://creativeco mmons.org/publicdomain/zero/1.0/) applies to the data made available in this article, unless otherwise stated in a credit line to the data. 
Keywords: Insulin sensitivity, Lipid profile, Strength, Physical function, Body composition, Rate of perceived exertion

\section{Introduction}

Type 2 diabetes (T2D) is one of the fastest growing chronic diseases and is a major burden on the global economy and healthcare system [8]. The International Diabetes Federation [20] predicted that more than half a billion people will have T2D by 2030 . It is well documented that exercise is essential in managing T2D, with evidence showing that performing structured aerobic and resistance exercise for more than 150 min per week can improve insulin sensitivity and glycemic control [7]. However, T2D is often associated with other comorbidities which may limit the individual's aerobic capacity, physical ability, and strength $[32,41]$ to perform physical activity. This may reduce exercise tolerance and adherence for patients with T2D to conventional exercise training [25]. Thus, developing an exercise intervention that is efficient and well tolerated for patients with T2D is vital.

Our daily activities consist of static (isometric), shortening (concentric) and lengthening (eccentric) muscle contractions (actions). Activities such as descending stairs, walking or running downhill, and lowering weights predominantly demand eccentric contractions in which contracting muscles are lengthened while resisting against load [27]. Muscles can generate more force maximally and can be loaded greater during eccentric than concentric contractions [17, 35]. Moreover, the metabolic demand of eccentric exercise is much less (25-50\%) than concentric exercise at the same workload [24]. Hence, eccentric exercises (e.g., descending stair walking) can be performed with reduced perceived effort when compared with concentric exercises (e.g., ascending stair walking) [25]. These characteristics make eccentric exercises advantageous and beneficial for less fit individuals such as patients with chronic diseases [27].

Importantly, several studies have shown that eccentric exercise training is more effective than concentric exercise training in improving insulin sensitivity, glycemic control, blood lipid profile, and physical fitness in healthy individuals $[4,5,10]$. For example, Chen et al. [4] reported that progressive eccentric resistance training of the knee extensors performed once a week for 12 weeks improved insulin sensitivity, lipid profile, physical functional performance, and muscular strength greater than concentric resistance training in healthy elderly men. Drexel et al. [10] showed that walking downhill for eight weeks 3-5 times a week significantly improved insulin action, glucose and lipid metabolism and body mass index. Similarly, a recent study demonstrated that descending stair walking performed three times a week for 12 weeks significantly improved body composition and insulin sensitivity in young women who are obese [5]. Marcus et al. [30] investigated a combination of aerobic and eccentric resistance training (AE/ECC) against aerobic only training (AE) performed three times a week for 16 weeks in patients with T2D. They found greater improvements in HbA1c levels for the AE/ECC (-0.59\%) than $\mathrm{AE}(-0.31 \%)$, and greater increases in thigh lean mass for the AE/ECC (15\%) than AE group (-6\%). Additionally, the six-minute walk test (6MWT) increased by $48 \%$ and body mass index (BMI) decreased by $2 \%$ for the $\mathrm{AE} / \mathrm{ECC}$ group only. These studies suggest that eccentric exercise may be beneficial not only in improving insulin sensitivity and glycemic control, but also improving physical function for people with T2D.

Previous eccentric exercise studies only used resistance exercise of an isolated single muscle group or aerobic mode of exercise as an intervention for patients with T2D [13]. To the best of our knowledge, no studies have investigated the effects of eccentric-only resistance training on insulin sensitivity, lipid profile, body composition, muscle strength and physical function in adults with T2D in comparison to concentric-only resistance training. Therefore, the primary aim of this study was to compare 12 -weeks of whole-body progressive eccentric-only and concentric-only resistance training to examine its effects on insulin sensitivity, glycemic control and blood lipid profile in adults with T2D. Secondary outcome measures included body composition, muscle strength, and physical function. Based on the results of a previous study [4], it was hypothesised that eccentric-only resistance training would be more effective than concentric-only resistance training in improving all outcome measures.

\section{Methods}

\section{Participants}

Fifty one adults who were diagnosed with T2D were screened, and 21 eligible participants were identified for this study (Fig. 1). The inclusion criteria were as follows: (1) adults aged 50-80 years, (2) fasting glucose $>7.0 \mathrm{mmol} / \mathrm{L}$ at the time of diagnosis, (3) no acute illness or any musculoskeletal, cardiovascular or neurological disorder that could inhibit exercise performance or put participants at risk from exercising, (4) no resistance training for at least 3 months prior to participation in the present study, and (5) medical clearance for participation. The participants provided written informed consent, and medical clearance gained from their general 


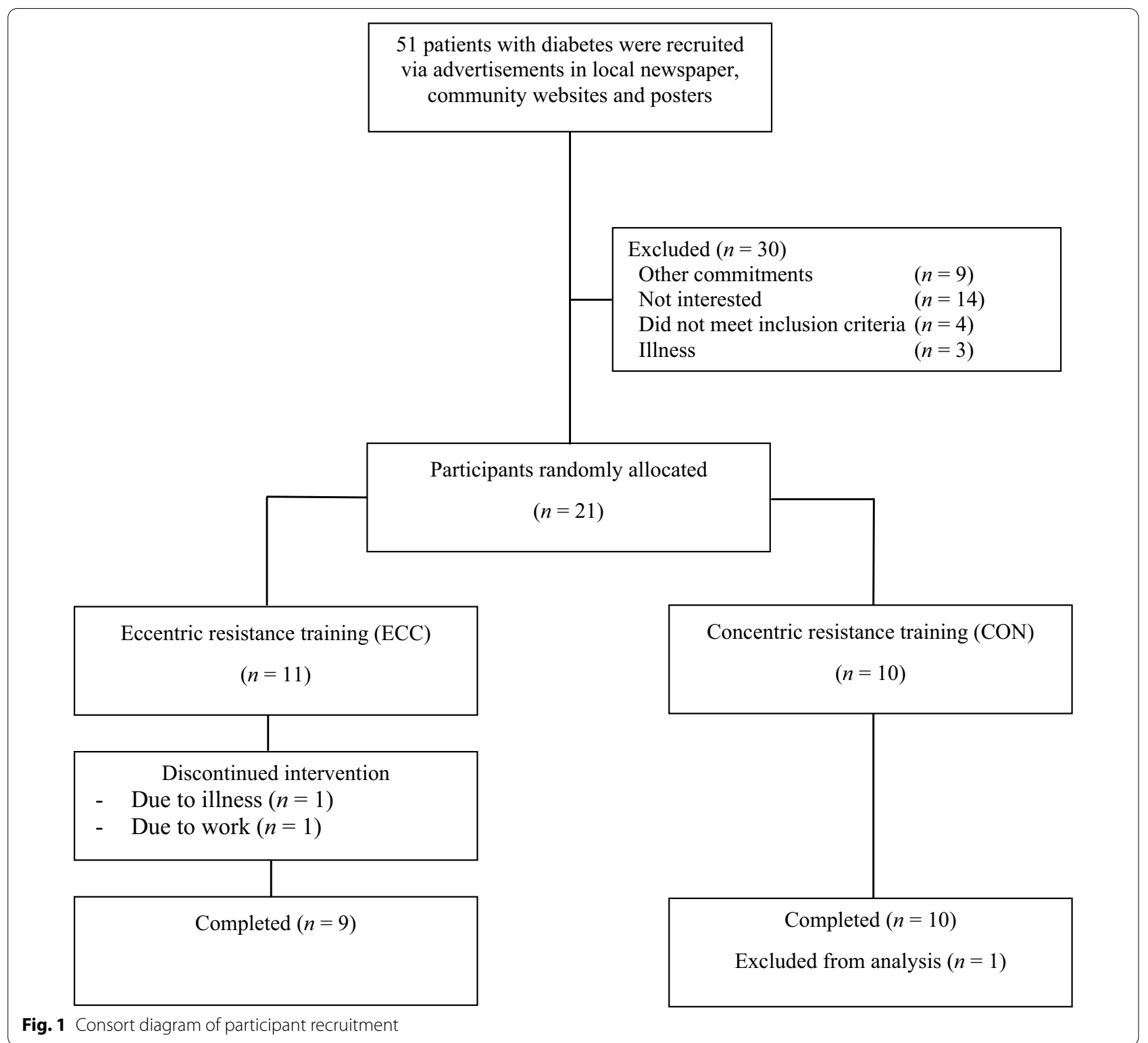

practitioner prior to participation in the study. They were instructed not to perform any resistance exercise or change their dietary habits during the experimental period. The study was approved by the Edith Cowan University Human Research Ethics Committee and was conducted in accordance with the Declaration of Helsinki.

Twenty one participants were stratified by age, gender, fasting glucose and previous physical activity level, and randomly allocated to either an ECC $(n=11)$ or a CON resistance training group $(n=10)$ as shown in Fig. 1. Two participants in the ECC group withdrew from the study due to illness or work commitment, and one participant in the CON group was excluded from the analysis, as this participant had much higher blood diabetes and lipid profile markers at baseline when compared with the rest of the participants. Thus, the final number of participants was nine for the ECC group [6 male, 3 female; mean $\pm S D$ (range) age $65.1 \pm 9.8(50-74)$ years, height $1.73 \pm 0.12(1.57-1.91)$ $\mathrm{m}$, body mass $90.4 \pm 16.3(62.0-110.0) \mathrm{kg}$, and BMI $\left.30.3 \pm 5.2(21.9-36.7) \mathrm{kg} / \mathrm{m}^{2}\right]$, and also nine for the CON group [7 male, 2 female; age: $63.2 \pm 8.6(50-79)$ years, height: $1.74 \pm 0.10(1.58-1.87) \mathrm{m}$, body mass: $89.5 \pm 15.3$ (75.1-108.3) kg, and BMI: $29.4 \pm 3.8$ (24.8$\left.33.7) \mathrm{kg} / \mathrm{m}^{2}\right]$. No significant differences between groups were found for the baseline measures. 


\section{Study design}

The exercise intervention period was 12 weeks, and testing was conducted at baseline (week 0), mid-intervention (week 6) and post-intervention (week 13). Prior to the commencement of the exercise intervention, the participants attended two separate sessions: (1) the first session to obtain baseline measurements (fasting blood test, body composition and anthropometric measurements) and (2) the second session was a familiarisation session and further baseline measures. During the familiarisation session, the investigator demonstrated correct form and technique of all eight exercises on the Cybex resistance machines (Cybex VRS, MA, USA). The participants were given the opportunity to perform all the exercises to ensure they were comfortable with the machines and familiarised themselves with the exercises with very light weight. These two sessions were conducted within oneweek prior to the first exercise training session.

The independent variable was the mode of exercise; eccentric-only versus concentric-only resistance exercise training as described below. The dependent variables consisted of blood biomarkers (insulin sensitivity, glycemic control and blood lipid profile), body composition, muscle strength, and physical function. Changes in these variables from pre- to post-intervention were compared between groups.

\section{Exercise intervention}

Participants performed supervised resistance exercises (chest press, lateral pulldown, bicep curl, triceps extension, abdominal crunch, leg extension, leg curl and calf raise) on Cybex resistance machines (Cybex VRS, MA, USA) targeting upper- and lower-body muscle groups twice a week on non-consecutive days for 12 weeks, based on the current recommended exercise guidelines for T2D [19]. A periodised and progressive resistance training program was adapted from a previous study [4], with 2-3 sets of 10 repetitions for each exercise for each session (Table 1). Chen et al. [4] found that a similar exercise protocol ( 3 or 6 sets $\times 10$ repetitions of the knee extensors) performed once a week for 12 weeks was effective for improving insulin sensitivity, glycemic control, and lipid profile in healthy older men, particularly in the eccentric group. The exercise load was based on the one-repetition maximal concentric strength (1-RMcon) measured at baseline for each exercise and gradually increased to $100 \%$ of 1-RMcon (Table 1). Due to increases in 1-RMcon throughout the 12 weeks, it should be noted that the $100 \%$ load was still submaximal. To minimise delayed onset muscle soreness particularly for the ECC group, the load was set at 10\% of 1-RMcon strength in week 1 and progressively increased every 2 weeks up to
Table 1 Resistance exercise training load and volume for the ECC group and CON group (CON) for 24 training sessions over 12 weeks

\begin{tabular}{|c|c|c|c|c|c|}
\hline \multirow[t]{2}{*}{ Week } & \multirow[t]{2}{*}{ Session } & \multicolumn{2}{|c|}{ Eccentric training } & \multicolumn{2}{|c|}{ Concentric training } \\
\hline & & Load & Volume & Load & Volume \\
\hline \multirow[t]{2}{*}{1 st } & 1 & $10 \%$ & $2 \times 10$ & $50 \%$ & $2 \times 10$ \\
\hline & 2 & & & & \\
\hline \multirow[t]{2}{*}{ 2nd } & 3 & $20 \%$ & $3 \times 10$ & $60 \%$ & $3 \times 10$ \\
\hline & 4 & & & & \\
\hline \multirow[t]{2}{*}{$3 \mathrm{rd}$} & 5 & $40 \%$ & $2 \times 10$ & $70 \%$ & $2 \times 10$ \\
\hline & 6 & & & & \\
\hline \multirow[t]{2}{*}{4 th } & 7 & $40 \%$ & $3 \times 10$ & $70 \%$ & $3 \times 10$ \\
\hline & 8 & & & & \\
\hline \multirow[t]{2}{*}{ 5th } & 9 & $60 \%$ & $2 \times 10$ & $80 \%$ & $2 \times 10$ \\
\hline & 10 & & & & \\
\hline \multirow[t]{2}{*}{ 6th } & 11 & $60 \%$ & $3 \times 10$ & $80 \%$ & $3 \times 10$ \\
\hline & 12 & & & & \\
\hline \multirow[t]{2}{*}{ 7th } & 13 & $75 \%$ & $2 \times 10$ & $90 \%$ & $2 \times 10$ \\
\hline & 14 & & & & \\
\hline \multirow[t]{2}{*}{ 8th } & 15 & $75 \%$ & $3 \times 10$ & $90 \%$ & $3 \times 10$ \\
\hline & 16 & & & & \\
\hline \multirow[t]{2}{*}{ 9th } & 17 & $90 \%$ & $2 \times 10$ & $95 \%$ & $2 \times 10$ \\
\hline & 18 & & & & \\
\hline \multirow[t]{2}{*}{ 10th } & 19 & $90 \%$ & $3 \times 10$ & $95 \%$ & $3 \times 10$ \\
\hline & 20 & & & & \\
\hline \multirow[t]{2}{*}{ 11th } & 21 & $100 \%$ & $2 \times 10$ & $100 \%$ & $2 \times 10$ \\
\hline & 22 & & & & \\
\hline \multirow[t]{2}{*}{ 12th } & 23 & $100 \%$ & $3 \times 10$ & $100 \%$ & $3 \times 10$ \\
\hline & 24 & & & & \\
\hline TWL (kg) & & 112,95 & $45,649.0$ & 148,83 & $3,131.0$ \\
\hline
\end{tabular}

Exercise intensity and training volume (number of sets $\times$ number of contractions) were progressively increased over the 24 sessions. The total weight lifted (TWL) over 24 training sessions for all eight exercises (mean $\pm S D$ ) is shown in the last row

$100 \%$ of 1-RMcon strength in week 12. Participants in the ECC group were instructed to lower the weight and resist against muscle lengthening actions to elicit eccentric contractions at a guided and slow controlled pace of 5 -s. For the CON group, the load increased from 50 to $100 \%$ of $1-$ RMcon strength over 24 sessions. Participants in the CON group were instructed to raise or lift the weights within 1-2 s to a fully extended or flexed position dependent on the exercise. Assistance was provided to lower or raise the weights to enable the participant to perform eccentric-only or concentric-only contractions. The rest period between repetitions was approximately 3 -s, and 60-s between sets for both ECC and CON. The Borg's CR-10 rating of perceived exertion (RPE) scale was used to assess the participant's effort immediately after each exercise and session. Muscle soreness was assessed $48-72 \mathrm{~h}$ after each session using a $10-\mathrm{cm}$ visual analog 
scale (VAS) anchored "0: no pain" and "10: maximal pain imaginable".

\section{Primary outcome measure Blood biomarkers}

Blood samples were obtained in the morning after a 10-h overnight fast at baseline, mid-intervention and post-intervention by a qualified phlebotomist. Venous blood samples were collected from a superficial vein on the radial aspect of the arm by a standard venipuncture, and drawn into the following vacutainer tubes: a 4-ml K2EDTA, a 6-ml fluoride oxalate and a 9-ml serum separator (SST) tube (Vacuette Tube, Greiner Bio-One, Austria). The SST sample was left to clot for $10 \mathrm{~min}$ at room temperature and all samples were centrifuged at $3000 \mathrm{rpm}$ for $10 \mathrm{~min}$ at $4{ }^{\circ} \mathrm{C}$ (Thermo Scientific Heraeus Multifuge 3S-R Centrifuge, Lagenselbold, Germany), aliquoted to several sampling tubes and stored in a $-80{ }^{\circ} \mathrm{C}$ alarmed controlled freezer (Thermo Scientific Forma 88000 Series Upright Freezer, Lagenselbold, Germany). The samples were analysed for plasma glucose, serum insulin, HbA1c, triglycerides, total cholesterol, high-density lipoprotein (HDL) and low-density lipoprotein (LDL). Insulin resistance was calculated using the updated homeostasis model assessment based on the following formula: [HOMA2-IR = fasting plasma glucose $(\mathrm{mmol} / \mathrm{L}) \times$ fasting serum insulin $(\mathrm{uU} / \mathrm{ml}) / 22.5]$.

\section{Secondary outcome measures}

\section{Body composition and anthropometry measurements}

Body composition was measured using a dual energy X-ray absorptiometry imaging scanner (Horizon DXA System, Hologic Inc., MA, USA) by a trained technician. Each participant was instructed to lie supine and still on a scanning bed with their palms faced down, feet position shoulder width apart and toes angled towards each other. The scanning process lasted approximately $3-4 \mathrm{~min}$. On completion, the scans were analysed using the Hologic QDR Software for Windows (Hologic, Bedford, MA, USA), which integrates whole-body measurement and standard body regions including the upper- and lowerlimb, and trunk, delineated by specific anatomical landmarks. Whole-body fat mass (FM) and lean mass (LM) in $\mathrm{kg}$, body fat percent (\%), and regional tissue composition were determined by manipulation of the segmental lines according to specific anatomical landmarks [44] with appendicular skeletal muscle mass (ASM) calculated from the sum of upper- and lower-limb LM [16].

Height and body mass were measured using a wallmounted stadiometer (Livingstone International Healthcare Pty Ltd, Australia) and a calibrated electronic weight scale (Model \#22089, SECA, Germany) to an accuracy of $0.5 \mathrm{~cm}$ and $0.1 \mathrm{~kg}$, respectively. BMI was calculated from the mass divided by the height in meters squared $(\mathrm{kg} /$ $\mathrm{m}^{2}$ ). The waist to hip ratio (WHR) was calculated from the measurements of waist circumference (between the lowest rib and iliac crest) and hip circumference (around the largest protrusion of the buttocks) using a flexible tape measure.

\section{Muscle strength}

Dynamic maximal muscle strength for all eight exercises was assessed at baseline, 6-week and post-intervention using 1-RMcon, which was the maximal weight that could be lifted once with correct technique. The protocol for assessing each participant's 1-RMcon was as follows; (1) each participant performed one set of six repetitions at approximately $60 \%$ of the participant's perceived maximal strength, (2) after two minutes of rest, the participant performed three repetitions at approximately $80 \%$ of the participant's perceived maximal strength, and (3) the load was gradually increased with 2 min rest between attempts until the participant failed to complete a full repetition with correct technique [43]. RPE was recorded after each attempt to ensure that the participant was able to tolerate the load and intensity. The load for 1-RMcon was recorded as the last successful attempt for each exercise. This measurement was also used to determine the individual exercise load for each participant.

\section{Physical function and balance}

A battery of physical function tests including a six-minute walk test (6MWT), 5-repetition chair rise test (CR), and 3-m timed up-and-go test (TUG) were performed at baseline and post-intervention. Three trials were performed for CR and TUG with one-minute rest between trials but the 6MWT was performed once.

The 6MWT is one of the most valid and common measures of physical functional capacity [14]. For the $6 \mathrm{MWT}$, a 20-m track was set up with cones at each end of an unobstructed corridor. Each participant was instructed to walk back and forth as quickly as possible for six minutes. A digital stopwatch (A601X Accusplit Pro Survivor Stopwatch, CA, USA) was used to monitor the time, with the investigator notifying the participant of the amount of time left at each minute. At the end of six minutes, the participant was requested to stop walking, and the distance was measured.

The CR test is widely used to measure lower limb strength and endurance [1]. For the CR test, a chair was positioned up against the wall and the participant was instructed to be seated with their back against the back rest, arms folded across the chest and feet positioned shoulder width apart on the floor. The participant was instructed to rise to a full standing position and sit back down with their back touching the back rest, repeating 
five times as quickly as possible. The fastest performance of the three trials was recorded for further analysis.

The TUG test is used to assess mobility and balance [15]. In the TUG test, a chair was placed up against the wall and the distance of three meters from the chair was marked with a cone. Each participant was instructed to be seated in a similar position as the CR test. The participant was instructed to rise from the chair (without using the hands for support), walk as fast as possible, turn around the cone positioned at the end of the route, return and sit back down on the chair with their back against the back rest. The fastest time of the three trials was recorded for further analysis.

A sensory organisation test (SOT) was performed on the Neurocom Smart Balancemaster (NeuroCom Balance Manager, WA, USA) to assess the participant's static and dynamic balance using various conditions including eyes open and eyes closed. The participant was required to stand as still as possible with both hands beside their body during each condition while the equilibrium score was quantified. The total score was reported between 0 to 100 . A score close to 100 indicates good stability and minimum sway whereas swaying to the limits of stability received a very low score. A score of 0 was automatically assigned to all falls or stopped trials.

\section{Statistical analysis}

The sample size was estimated by a-priori power analysis using G*Power (http://www.gpower.hhu.de/). Based on the effect size of 0.25 for a possible difference in the changes in fasting insulin and glucose concentrations in the blood between the two exercise groups from a previous study [4], with the $\alpha$-level of 0.05 and a statistical power $(1-\beta)$ of 0.80 , it was found that at least 16 participants were required for this study. All statistical analyses were performed using the IBM SPSS v27.0 statistical package (SPSS Inc., Chicago, IL, USA). Data was assessed by a Shapiro-Wilk test for normality and a Levene test for the homogeneity of variance assumption. Baseline values of each variable were compared between ECC and $\mathrm{CON}$ groups by an independent samples t-test. Changes in each dependent variable over time were compared between the ECC and CON groups by a repeated measures mixed-design two-way ANOVA. When a significant interaction (group $\times$ time) effect was found, a Tukey's post-hoc test was performed to identify and compare the differences between groups for each time point. The changes in each dependent variable from pre- to posttraining were also compared between groups by independent t-test. Effect sizes (ES) was calculated using Cohen's $d$ represented as $E S=\left(\right.$ Mean $_{\text {Post }}-$ Mean $\left._{\text {Pre }}\right) /$ $\mathrm{SD}_{\text {Pre }}$ and was considered as small $(d>0.2)$, medium $(d>0.5)$, and large effect $(d>0.8)$ [6]. All statistical results are shown in mean \pm standard deviation (SD) unless otherwise stated.

\section{Results}

The participants completed all 24 sessions of the exercise intervention as planned. Both groups completed the same number of total muscle contractions (600 contractions) for each of the eight exercises over the 12-week period. The total weight lifted was $112,955.0 \pm 45,649.0 \mathrm{~kg}$ for the ECC group and 148,832.0 $\pm 43,131.0 \mathrm{~kg}$ for the CON group, without a significant difference between groups (Table 1).

Sessional RPE increased over 24 sessions as the exercise load gradually increased for both groups (Fig. 2). On average, the CON group elicited a higher RPE $(5.4 \pm 1.1)$ than the ECC group $(2.9 \pm 1.2)$. At week 12 of the intervention, both groups performed 3 sets of 10 repetitions at a load of 100\% 1-RMcon. The average sessional RPE was $4.1 \pm 2.1$ for the ECC group, and $7.6 \pm 2.7$ for the CON group, with a significant difference between groups.

Minimal muscle soreness (VAS $<20 \mathrm{~mm}$ ) in both groups were observed 24-48-h after several exercise sessions and even after the higher-intensity sessions. No significant difference was found between groups.

\section{Blood biomarkers}

As shown in Table 2, no significant difference between groups was found for any of the blood markers at baseline. Significant changes $(p<0.05)$ including increases in fasting serum insulin $(2.7 \pm 1.3 \mathrm{mU} / \mathrm{L})$ and HOMA2IR $(0.4 \pm 0.1)$, and decreases in HbA1c $(-0.4 \pm 0.1 \%)$ were evident after 6 weeks of training in the ECC group only. HbA1c significantly decreased $(p<0.05)$ after 12 weeks of concentric training $(-0.3 \pm 0.0 \%)$ but not in the ECC group $(-0.1 \pm 0.1 \%)$. No significant changes were found in lipid profile for both groups from pre- to post-intervention.

\section{Body composition and anthropometry measurements}

Significant reductions in FM (ECC $-2.1 \pm 1.3 \mathrm{~kg}$, $p=0.025 ; \mathrm{CON}-2.2 \pm 1.2 \mathrm{~kg}, p=0.005)$ and body fat $\% \quad($ ECC $-2.0 \pm 0.6 \%, p=0.017 ;$ CON $-2.3 \pm 0.1 \%$, $p=0.001)$ were found from pre- to post-intervention; however, no significant differences were evident between groups (Table 3 ). Total LM increased significantly after training for both ECC $(1.8 \pm 0.8 \mathrm{~kg}, p=0.034)$ and CON $(2.0 \pm 0.3 \mathrm{~kg}, p=0.015)$ groups.

As shown in Table 3 , none of the anthropometry measures were significantly different between groups at baseline. Significant decreases $(p<0.05)$ in waist circumference were found for both ECC $(-5.2 \pm 1.0 \mathrm{~cm})$ and CON $(-4.3 \pm 0.8 \mathrm{~cm})$ groups from pre- to post-intervention. A significant decrease in hip circumference was 


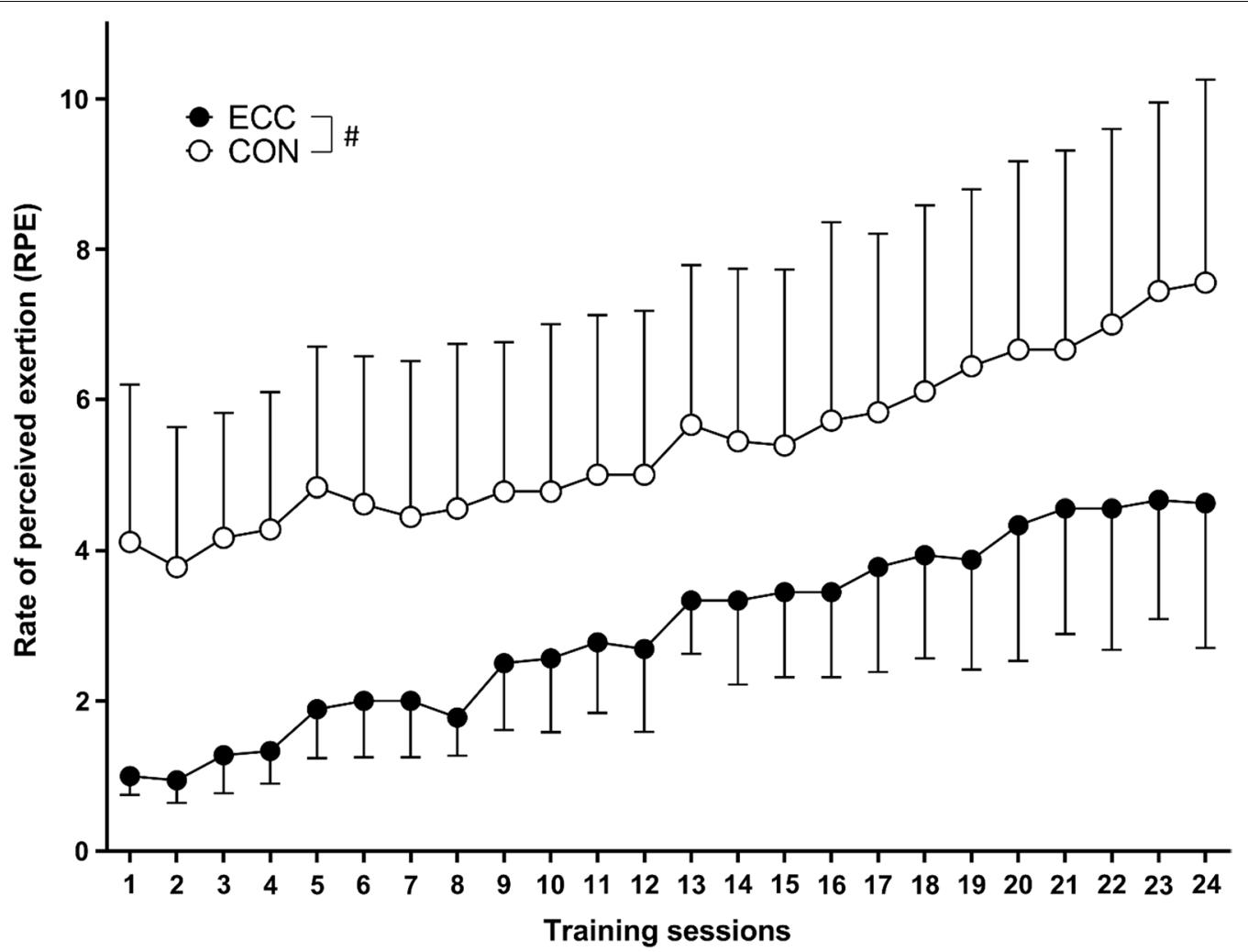

Fig. 2 Changes (mean $\pm S D$ ) in the average sessional RPE between the ECC and CON groups recorded after each exercise training session for 12 weeks ( 24 sessions). Significant $(p<0.05)$ difference between ECC and CON for all sessions

found for the CON group only $(-4.8 \pm 0.6 \mathrm{~cm})$, and a significant decrease in the waist-to-hip ratio was evident for the ECC group only $(-0.038 \pm 0.002)$ from pre- to post-intervention.

\section{Muscle strength}

No significant difference between groups was found for any of the baseline muscle strength measures. Significant increases were found in upper- and lower-body strength $(p<0.05)$ for all eight exercises in both groups (Fig. 3). Upper-body strength increased between $12-33 \%$ in the ECC group and $27-43 \%$ in the CON group, while lowerbody strength increased between $32-37 \%$ in the ECC group and $34-68 \%$ in the CON group. Significant differences were found between the ECC and CON groups for bicep curl, calf raise and abdominal crunch exercises $(p<0.05)$ with the CON group showing greater increases.

\section{Physical function and balance}

The baseline values were not significantly different between groups for physical function and balance measures (Table 4). After 12 weeks of training, both groups showed improvements $(p<0.05)$ for 6MWT distance
(ECC $56.8 \pm 2.2 \mathrm{~m}, \mathrm{CON} 63.4 \pm 12.0 \mathrm{~m}$ ) and CR time $(\mathrm{ECC}-1.8 \pm 1.3 \mathrm{~s}, \mathrm{CON}-2.3 \pm 1.6 \mathrm{~s})$ without significant differences between groups. Significant improvements for TUG were found only for the ECC group $(-0.8 \pm 0.2 \mathrm{~s})$. No significant changes before and after the 12-week training intervention were found for both groups in the balance tests including the overall score (Table 4).

\section{Discussion}

To the best of our knowledge, this was the first study to compare the effects of progressive eccentric-only versus concentric-only resistance exercise training on biochemical and physical outcome measures in patients with T2D. No significant changes were found in blood diabetes and lipid profile markers at the end of the intervention for both training groups (Table 2). However, significant improvements in body composition (Table 3), muscle strength (Fig. 3) and physical function (Table 4) were observed after 12 weeks of both eccentric-only and concentric-only resistance training. Thus, the hypothesis that eccentric-only resistance training would improve all outcome measures better than concentric-only resistance training was not supported by the results. However, it 


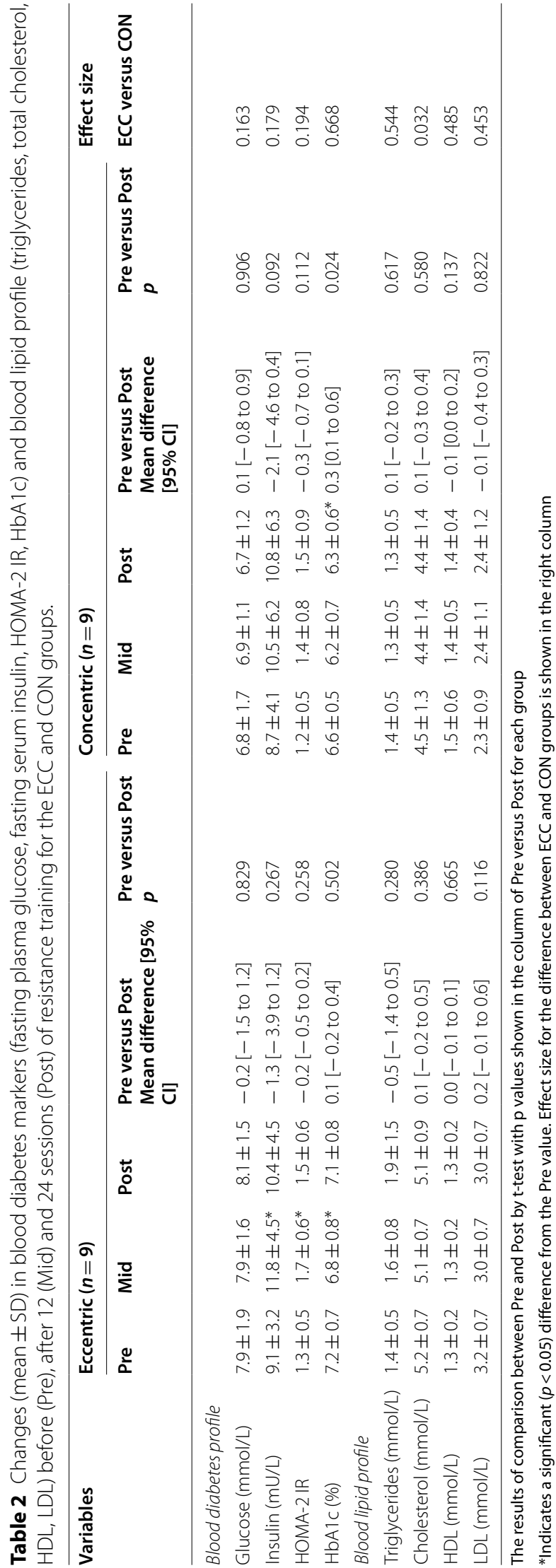


Table 3 Changes (mean \pm SD) in anthropometry measures, fat mass and lean mass from baseline (Pre) to post-intervention (Post) for ECC and CON groups

\begin{tabular}{|c|c|c|c|c|c|c|c|c|c|}
\hline \multirow[t]{2}{*}{ Variables } & \multicolumn{4}{|c|}{ Eccentric $(n=9)$} & \multicolumn{4}{|c|}{ Concentric $(n=9)$} & \multirow{2}{*}{$\begin{array}{l}\text { Effect size } \\
\text { ECC versus } \\
\text { CON }\end{array}$} \\
\hline & Pre & Post & $\begin{array}{l}\text { Pre versus } \\
\text { Post } \\
\text { Mean } \\
\text { difference } \\
{[95 \% \mathrm{Cl}]}\end{array}$ & $\begin{array}{l}\text { Pre versus } \\
\text { post } \\
p\end{array}$ & Pre & Post & $\begin{array}{l}\text { Pre versus } \\
\text { Post } \\
\text { Mean } \\
\text { difference } \\
{[95 \% \mathrm{Cl}]}\end{array}$ & $\begin{array}{l}\text { Pre versus } \\
\text { Post } \\
p\end{array}$ & \\
\hline \multicolumn{10}{|c|}{ Anthropometry measures } \\
\hline $\begin{array}{l}\text { Body mass } \\
(\mathrm{kg})\end{array}$ & $90.4 \pm 16.3$ & $90.5 \pm 15.5$ & $\begin{array}{l}-0.1[-1.6 \\
\text { to } 1.4]\end{array}$ & 0.934 & $89.5 \pm 15.3$ & $89.4 \pm 14.2$ & $\begin{array}{l}0.1[-1.3 \text { to } \\
1.5]\end{array}$ & 0.861 & 0.070 \\
\hline $\begin{array}{l}\text { Body mass } \\
\text { index }(\mathrm{kg} / \\
\left.\mathrm{m}^{2}\right)\end{array}$ & $30.3 \pm 5.2$ & $30.2 \pm 4.7$ & $\begin{array}{l}0.1[-0.4 \text { to } \\
0.5]\end{array}$ & 0.786 & $29.4 \pm 3.8$ & $29.5 \pm 3.3$ & $\begin{array}{l}-0.1[-0.7 \\
\text { to } 0.4]\end{array}$ & 0.568 & 0.270 \\
\hline Waist (cm) & $104.4 \pm 14.3$ & $99.2 \pm 13.3^{*}$ & $\begin{array}{l}5.2[2.9 \text { to } \\
7.5]\end{array}$ & 0.001 & $99.28 \pm 12.07$ & $95.0 \pm 11.31^{*}$ & $\begin{array}{l}4.2[1.5 \text { to } \\
7.1]\end{array}$ & 0.008 & 0.198 \\
\hline Hip (cm) & $104.5 \pm 9.42$ & $103.17 \pm 8.40$ & $\begin{array}{l}1.3[-0.5 \text { to } \\
3.2]\end{array}$ & 0.132 & $105.17 \pm 11.3$ & $100.4 \pm 10.69^{*}$ & $\begin{array}{l}4.7[2.0 \text { to } \\
7.4]\end{array}$ & 0.004 & 1.174 \\
\hline $\begin{array}{l}\text { Waist-to-hip } \\
\text { ratio }\end{array}$ & $0.997 \pm 0.079$ & $0.960 \pm 0.077^{*}$ & $\begin{array}{l}0.0[0.0 \text { to } \\
0.0]\end{array}$ & 0.036 & $0.945 \pm 0.072$ & $0.948 \pm 0.078$ & $\begin{array}{l}0.0[0.0 \text { to } \\
0.0]\end{array}$ & 0.862 & 0.840 \\
\hline \multicolumn{10}{|l|}{ Fat mass (kg) } \\
\hline Total body & $33.6 \pm 10.6$ & $31.5 \pm 9.3^{*}$ & $\begin{array}{l}2.0[0.3 \text { to } \\
3.7]\end{array}$ & 0.025 & $30.7 \pm 7.6$ & $28.5 \pm 6.4^{*}$ & $\begin{array}{l}2.2[0.9 \text { to } \\
3.5]\end{array}$ & 0.005 & 0.545 \\
\hline Upper-limb & $3.7 \pm 1.2$ & $3.5 \pm 1.1$ & $\begin{array}{l}0.2[0.0 \text { to } \\
0.4]\end{array}$ & 0.090 & $3.5 \pm 0.8$ & $3.1 \pm 0.7^{*}$ & $\begin{array}{l}0.4[0.2 \text { to } \\
0.6]\end{array}$ & 0.003 & 0.982 \\
\hline Lower-limb & $10.5 \pm 3.2$ & $9.9 \pm 2.9^{*}$ & $\begin{array}{l}0.6[0.1 \text { to } \\
1.1]\end{array}$ & 0.024 & $9.5 \pm 2.8$ & $9.1 \pm 2.4$ & $\begin{array}{l}0.4[-0.1 \text { to } \\
0.8]\end{array}$ & 0.115 & 0.281 \\
\hline Trunk & $18.0 \pm 6.9$ & $16.8 \pm 6.1$ & $\begin{array}{l}-0.7[-1.4 \\
\text { to } 0.0]\end{array}$ & 0.092 & $16.4 \pm 4.7$ & $15.1 \pm 3.6^{*}$ & $\begin{array}{l}1.0[0.2 \text { to } \\
2.6]\end{array}$ & 0.029 & 0.259 \\
\hline Body fat (\%) & $36.6 \pm 8.6$ & $34.6 \pm 8.0^{*}$ & $\begin{array}{l}2.0[0.5 \text { to } \\
3.5]\end{array}$ & 0.017 & $34.1 \pm 6.5$ & $31.8 \pm 6.4^{*}$ & $\begin{array}{l}2.3[1.3 \text { to } \\
3.3]\end{array}$ & 0.001 & 0.371 \\
\hline \multicolumn{10}{|c|}{ Lean mass (kg) } \\
\hline Total body & $55.0 \pm 10.6$ & $56.8 \pm 11.4^{*}$ & $\begin{array}{l}1.8[3.3 \text { to } \\
0.2]\end{array}$ & 0.034 & $57.0 \pm 11.0$ & $59.0 \pm 11.3^{*}$ & $\begin{array}{l}2.0[-3.6 \text { to } \\
-0.5]\end{array}$ & 0.015 & 0.148 \\
\hline Upper-limb & $5.5 \pm 1.5$ & $6.0 \pm 1.8^{*}$ & $\begin{array}{l}0.6[1.7 \text { to } \\
0.5]\end{array}$ & 0.047 & $6.2 \pm 1.5$ & $6.3 \pm 1.6$ & $\begin{array}{l}0.1[-2.0 \text { to } \\
0.0]\end{array}$ & 0.638 & 0.806 \\
\hline Lower-limb & $16.8 \pm 3.5$ & $17.4 \pm 4.3$ & $\begin{array}{l}0.5[-1.0 \text { to } \\
0.0]\end{array}$ & 0.261 & $17.7 \pm 3.5$ & $18.6 \pm 4.1$ & $\begin{array}{l}0.1[-0.5 \text { to } \\
0.4]\end{array}$ & 0.070 & 0.204 \\
\hline Trunk & $29.6 \pm 5.7$ & $30.3 \pm 5.5$ & $\begin{array}{l}0.7[-1.4 \text { to } \\
0.0]\end{array}$ & 0.063 & $30.0 \pm 6.2$ & $31.0 \pm 5.5^{*}$ & $\begin{array}{l}1.0[-2.0 \text { to } \\
0.0]\end{array}$ & 0.037 & 0.395 \\
\hline $\begin{array}{l}\text { Appendicu- } \\
\text { lar skeletal } \\
\text { mass }\end{array}$ & $22.3 \pm 4.9$ & $23.5 \pm 6.0$ & $\begin{array}{l}1.1[-2.6 \text { to } \\
0.4]\end{array}$ & 0.119 & $23.8 \pm 4.9$ & $24.9 \pm 5.6$ & $\begin{array}{l}1.0[-2.2 \text { to } \\
0.0]\end{array}$ & 0.064 & 0.107 \\
\hline
\end{tabular}

The results of comparison between Pre and Post by t-test with $p$ values shown in the column of Pre versus Post for each group

*Indicates a significant $(p<0.05)$ difference from the Pre value. Effect size for the difference between ECC and CON groups is shown in the right column

should be noted that the average RPE during training was less for ECC $(2.9 \pm 1.2)$ than CON $(5.4 \pm 1.1)$ as shown in Fig. 2. It is important to note that the effects of resistance training on the outcome measures were induced by less strenuous exercise sessions in the eccentric-only than concentric-only training.

As shown in Fig. 2, the average RPE in the training sessions was "hard" for the CON group but "somewhat moderate" for the ECC group. The ECC group reported an average RPE of $4.1 \pm 2.1$ (moderate) while the CON group's RPE was $7.6 \pm 2.7$ (very hard) after performing 3 sets of 10 repetitions at $100 \%$ of baseline 1-RMcon during sessions $21-24$. This was in line with the findings of previous studies showing that eccentric exercise was easier to perform than concentric exercise for the same absolute workload [11, 26, 37, 38]. Importantly, minimal or no muscle soreness was reported after any exercise session in both groups even at high-intensity sessions. This was also evident in a previous study [4] and is likely due to the protective effect conferred by progressive overload which 


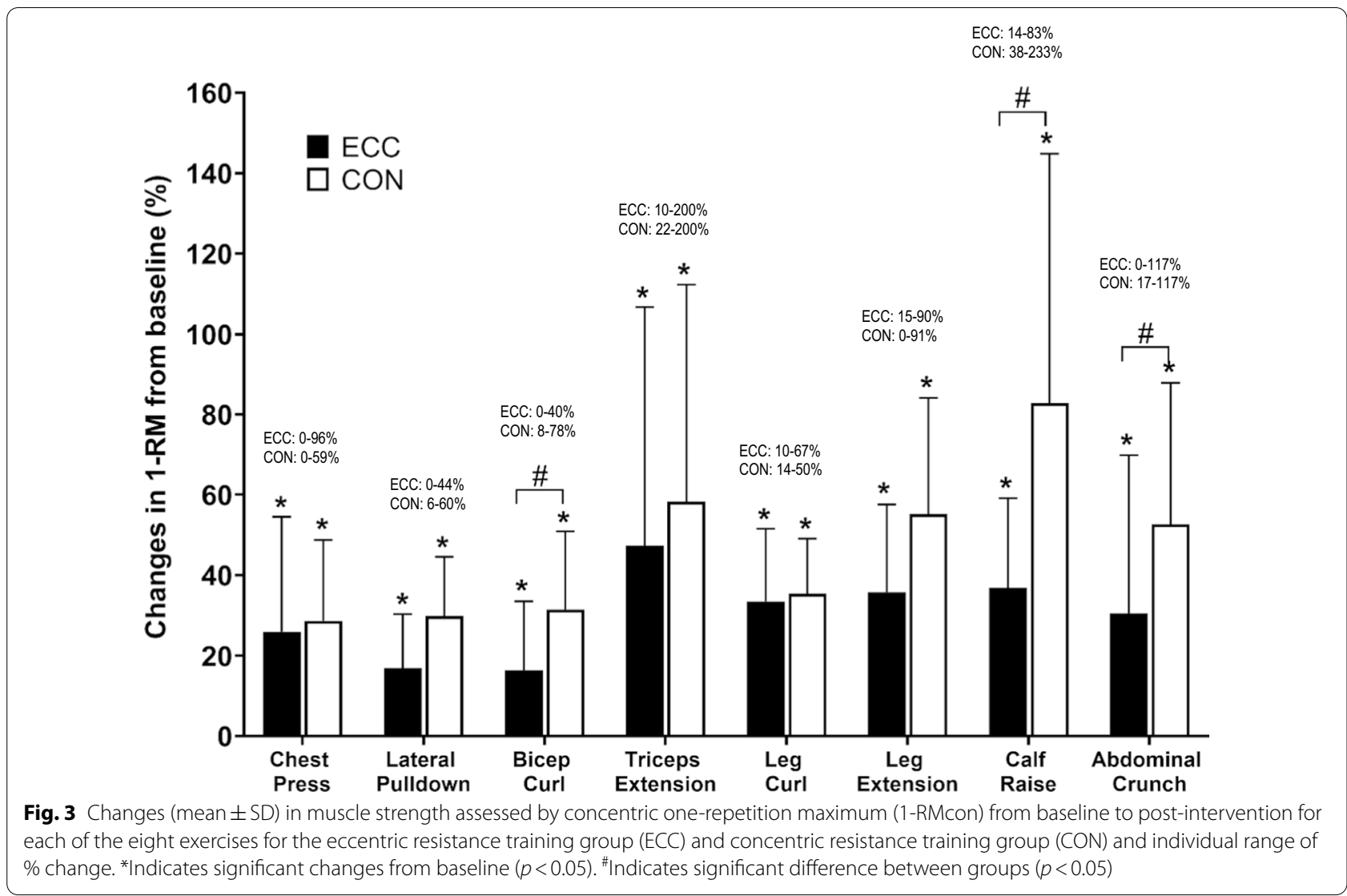

Table 4 Changes (mean \pm SD) in physical function assessed by six-minute walk test, five repetition chair rise time and 3-m timed up-and-go, and balance sensory measures for the total score, eyes closed and sway vision from baseline (Pre) to post-intervention (Post) for ECC and CON groups

\begin{tabular}{|c|c|c|c|c|c|c|c|c|c|}
\hline \multirow[t]{2}{*}{ Variables } & \multicolumn{4}{|c|}{ Eccentric $(n=9)$} & \multicolumn{4}{|c|}{ Concentric $(n=9)$} & \multirow{2}{*}{$\begin{array}{l}\text { Effect size } \\
\text { ECC versus } \\
\text { CON }\end{array}$} \\
\hline & Pre & Post & $\begin{array}{l}\text { Pre versus } \\
\text { post mean } \\
\text { difference } \\
{[95 \% \mathrm{Cl}]}\end{array}$ & $\begin{array}{l}\text { Pre versus } \\
\text { post } \\
p\end{array}$ & Pre & Post & $\begin{array}{l}\text { Pre versus } \\
\text { post } \\
\text { Mean } \\
\text { difference } \\
{[95 \% \mathrm{Cl}]}\end{array}$ & $\begin{array}{l}\text { Pre versus } \\
\text { post } \\
p\end{array}$ & \\
\hline \multicolumn{10}{|c|}{ Physical function } \\
\hline $\begin{array}{l}\text { 6-min walk } \\
\text { test (m) }\end{array}$ & $463.9 \pm 94.1$ & $520.7 \pm 91.9^{*}$ & $\begin{array}{l}-46.8[-98.1 \\
\text { to } 4.6]\end{array}$ & 0.018 & $507.9 \pm 79.9$ & $571.3 \pm 67.9^{*}$ & $\begin{array}{l}-59.7[-95.9 \\
\text { to }-23.5]\end{array}$ & 0.005 & 0.083 \\
\hline $\begin{array}{l}\text { 5-rep chair } \\
\text { rise (s) }\end{array}$ & $13.4 \pm 5.1$ & $11.6 \pm 3.8^{*}$ & $1.8[0.4$ to 3.1$]$ & 0.015 & $11.5 \pm 3.0$ & $9.2 \pm 1.4^{*}$ & $2.3[0.4$ to 4.3$]$ & 0.023 & 0.463 \\
\hline $\begin{array}{l}\text { 3-m timed-up } \\
\text { and go (s) }\end{array}$ & $7.1 \pm 2.2$ & $6.3 \pm 1.9^{*}$ & $0.6[0.0$ to 1.2$]$ & 0.005 & $5.8 \pm 0.8$ & $5.3 \pm 0.5$ & $0.5[0.0$ to 1.0$]$ & 0.055 & 0.014 \\
\hline \multicolumn{10}{|c|}{ Balance sensory measures } \\
\hline $\begin{array}{l}\text { Total compos- } \\
\text { ite score }\end{array}$ & $71.3 \pm 11.1$ & $76.3 \pm 6.3$ & $\begin{array}{l}-5.0[-11.0 \\
\text { to } 1.0]\end{array}$ & 0.089 & $74.3 \pm 6.6$ & $77.6 \pm 5.5$ & $\begin{array}{l}-3.2[-8.0 \\
\text { to } 1.6]\end{array}$ & 0.162 & 0.325 \\
\hline Eyes closed & $46.4 \pm 20.7$ & $63.2 \pm 10.1$ & $\begin{array}{l}-16.9[-35.1 \\
\text { to } 1.3]\end{array}$ & 0.065 & $57.9 \pm 11.1$ & $62.6 \pm 9.6$ & $\begin{array}{l}-4.7[-16.0 \\
\text { to } 6.6]\end{array}$ & 0.369 & 0.481 \\
\hline Sway vision & $50.3 \pm 27.7$ & $58.1 \pm 18.2$ & $\begin{array}{l}-7.9[-18.9 \\
\text { to } 3.1]\end{array}$ & 0.137 & $54.8 \pm 13.6$ & $61.8 \pm 10.2$ & $\begin{array}{l}-7.0[-18.6 \\
\text { to } 4.6]\end{array}$ & 0.201 & 0.493 \\
\hline
\end{tabular}


commenced from a very low intensity. This is important as muscle soreness after eccentric exercise can be a factor that discourages people from continuing regular exercise [18]. Using the progressive training protocol, the participants appeared to be able to adhere to the exercise training program over 12 weeks and achieved significant gains in muscle strength.

As shown in Table 2, the present study did not find any significant changes in blood diabetes markers following eccentric resistance training. Chen et al. [4] showed significant improvements in insulin sensitivity including reductions in fasting glucose, insulin, HOMA and HbA1c following 12 weeks of eccentric resistance training of the knee extensors in healthy older men. In the present study, the ECC group showed improvements in HbA1c after 6 weeks but not after 12 weeks of training. This difference could be attributed to prescribed medications consumed, which was recorded but not controlled in the present study. The effects of ECC resistance training on individuals with prescribed T2D medications (including the commonly prescribed Metformin) remains to be determined [34]. Muscle strength gains, increases in insulin sensitivity and improvements in glycemic control are considered normal adaptations to exercise training; however, some studies have found that Metformin inhibits muscle hypertrophy and blunts the effect of muscle mass gains in response to progressive resistance training in older adults [2, 28, 45]. The combination of Metformin and exercise may be less effective in reducing glycemic response [3] and may in fact attenuate the well-documented benefits of exercise alone [42]. In fact, Sharoff et al. [42] reported that exercise alone increased insulin sensitivity; however, a combination of exercise and Metformin did not enhance insulin sensitivity and showed an increase in glucose production due to less AMP-activated protein kinase (AMPK) activation. It is also possible that the effects of the exercise training on insulin sensitivity markers were masked by the effects of Metformin. Eighty percent of the participants in this study took Metformin as a prescribed diabetes medication which may explain the lack of significant changes in insulin sensitivity and glycemic control.

Paschalis et al. [33] found favorable changes in lipid profile including decreases in total cholesterol levels $(-8.8 \%)$, triglycerides $(-12.8 \%)$ and LDL $(-16.4 \%)$ after performing eccentric resistance training once a week for 8 weeks in a group of healthy women. Chen et al. [4] also showed significant changes in blood lipid markers (TC $-8 \%$, TG $-16 \%$, LDL $-8 \%$ ) after 12 sessions of knee extensor eccentric training over 12 weeks. However, the present study did not find any significant changes in blood lipid profile following ECC or CON training. The average fasting cholesterol levels were $5.6 \mathrm{mmol} / \mathrm{L}$ and
$4.5 \mathrm{mmol} / \mathrm{L}$ respectively for the ECC and CON groups. Critically, participants in this study had near-normal levels of lipid profile parameters observed at baseline; thus, the ceiling effect might explain no positive changes in blood lipid profile.

Both the ECC and CON groups showed a significant increase of approximately $3 \%$ in whole-body LM after 12 weeks of training (Table 3). Marcus et al. [30] reported an increase of up to $10.5 \%$ in thigh lean mass and decrease of $-1.2 \mathrm{~cm}$ around the thigh-intramuscular fat cross-sectional area using magnetic resonance imaging (MRI) scans after 16 weeks of high-force eccentric resistance exercises in combination with aerobic exercise in adults with T2D. Importantly, segmental LM significantly increased in the upper limb (9.1\%), lower limb (3.6\%) and abdominal (2.4\%) regions for the ECC group only (Table 3). Simultaneous gains in LM and decrease in body fat $\%$ are important in attenuating muscle wastage in the elderly [31]. In the present study, both groups demonstrated significant decreases in body fat \% including reductions in trunk fat, waist circumference, and total FM. We found a significant decrease of $2.1 \mathrm{~kg}$ in total body mass in the ECC group, with majority of the fat loss around the abdominal region. Julian et al. [22] observed decreases in whole-body $(-10 \%)$ and leg FM $(-6.5 \%)$ following 12 weeks of eccentric cycling training in obese adolescents, and stated that these improvements could be due to large increases in post-training resting energy expenditure after eccentric exercise. Gluchowski et al. [12] also found that eccentric exercise prescription was beneficial in improving body composition and could potentially be an important stimulus in preventing sarcopenia, osteoporosis, and obesity. However, the specific differences between eccentric and concentric muscle contractions remain unclear and require further investigation.

Eccentric exercise training increases muscle strength with lesser perceived effort when compared to concentric exercise [21]. Results from a meta-analysis showed that high-intensity eccentric resistance exercise was superior to concentric resistance exercise in stimulating muscle strength increases. This is possibly due to the higher force developed during eccentric contractions contributed through neurological, physiological and architectural changes [39]. Older adults seem to preserve greater residual force enhancement after eccentric contractions and can produce eccentric strength more efficiently than isometric and concentric strength, which may be a contributing factor to improvements in muscle strength [36]. Chen et al. [4] compared the effects of eccentric and concentric knee extension exercise performed once a week for 12 weeks and found greater increase in 1-RMcon strength in the eccentric group (49\%) than the concentric 
group (35\%). In contrast, the present study found that 1-RMcon knee extension strength had smaller increases for the ECC (36\%) than CON training (55\%) over 12 weeks (24 sessions). Due to the large variability among the participants for changes in the 1-RMcon strength, no significant difference between ECC and CON was detected for knee extension strength. Although the CON group commenced exercise load at $50 \%$ of 1 -RMcon in comparison to the ECC group at $10 \%$, the average total weight lifted over 24 sessions was not significantly different between CON and ECC groups. When comparing ECC and CON for each exercise, significant differences in the total weight lifted were found for the triceps extension only $(C O N>E C C)$. It is important to note that the increases in 1-RMcon strength was larger in the CON group for bicep curl, calf raise and abdominal crunch exercises than the ECC group. The magnitude of changes in 1-RMcon strength varied among the exercises such that the largest increase was found for calf raise (ECC $37 \%$, CON 68\%) and the smallest increase was found for bicep curl (ECC 11\%, CON 27\%). It should also be noted that the 1-RM measurement was performed concentrically; thus, participants in the CON group might have advantages due to the specificity principle. Future studies could potentially include other strength measures such as maximal voluntary isometric and/or isokinetic contraction strength to measure force and velocity.

Following the 12-week intervention, participants exhibited improvements in physical function demonstrated by faster times recorded for the CR (ECC 13.4\%, CON 20.0\%) and TUG tests (ECC 11.3\%, CON 8.6\%), and increased distance for the 6MWT (ECC 12.2\%, CON $12.5 \%$ ) when compared with the baseline values (Table 2). Our results were consistent with the findings of previous studies $[9,37]$ reporting that eccentric resistance exercise was efficient and effective in improving functional capacity in older adults. Raj et al. [37] reported similar improvements in functional performance after 16-weeks of eccentrically-biased resistance training $(6 \mathrm{MWT} 7 \%$, TUG 3\%) and conventional resistance training (6MWT $5 \%$, TUG 5\%) consisting of leg press, toe press, bench press and lateral pulldown. Dias et al. [9] reported significant improvements in 6MWT (12\%), CR (15\%) and TUG (16\%) after 6 weeks of eccentric-focused resistance training consisting of leg press, seated row, knee extension and bench press, although the leg press 1-RMcon did not change significantly throughout the study. It appears that the hamstring muscle is a vital muscle in walking ability as it controls and stabilises the hip movement. The degree of hip and knee flexion appears to increase to maintain the body's center of gravity as walking speed increases [29]. This is important in maintaining physical functional performance and balance in adults with chronic disease.
The balance ability assessed by SOT did not show significant changes after ECC or CON training (Table 2). Previous studies $[4,23]$ showed greater improvements in balance after eccentric than concentric resistance training using different tests (Berg balance test, static balance test with eyes open and closed). It is important to note that all the participants achieved scores above $80 \%$ during their baseline testing and this ceiling effect may be the reason for no further improvement found in this study [40].

There were several limitations in the present study, which should be considered for future studies. Firstly, the sample size was small as many participants recruited for the study did not want to commit to three months of continuous training. Secondly, no control group without any exercise intervention was included in the present study. Although, it is reasonable to assume that no changes in the outcome measures would have been found for the control group. Thirdly, the participants were asked to record a 3-day food diary; however, they were not required to restrict their caloric intake or other physical activities during the intervention. Even though the participants were recommended not to change their eating, drinking or physical activity habits, additional lifestyle and behavioral changes due to the positive effects of participating in an exercise intervention could not be entirely ruled out. Fourthly, the exercise protocol of the present study required the investigators to physically assist and lift heavy loads to eliminate the eccentric or concentric phase. The practical applications of performing eccentric only resistance exercises must be considered, as specific eccentric-only equipment is costly and may not be readily available in a clinical setting. It should also be noted that the exercise intensity and the volume were not matched between the eccentric and concentric groups. It is possible to increase the load for the eccentric-only exercises, since maximal voluntary contraction strength is greater for eccentric than concentric contractions. Future studies should investigate if eccentric and concentric resistance training of the same absolute workload, volume and/or RPE at an increased frequency can elicit greater improvements in blood markers for the eccentric-only than concentric-only training.

\section{Conclusion}

In summary, no significant changes in blood biomarkers were found after the 12-week intervention and no significant differences were found between the two groups; thus, our hypotheses were not supported. Nevertheless, there were some noteworthy findings in the present study. All participants were able to tolerate and perform the exercises with minimal muscle soreness and no injury, contraindications, or adverse events. 
Our findings demonstrated that commencing eccentric resistance training at a lower intensity with gradual progression can elicit significant exercise training adaptations and positive outcomes in improving body composition, muscle strength and physical function for adults with T2D. This is important to prevent physical decline and sarcopenia and improve overall quality of life particularly for older adults who have limited functional capacity and clinical health conditions.

\begin{abstract}
Abbreviations
1-RM: One-repetition maximum; 6MWT: Six-minute walk test; ANOVA: Analysis of variance; ASM: Appendicular skeletal muscle mass; BMI: Body mass index; CON: Concentric resistance training; CR: Chair rise; ECC: Eccentric resistance training; ES: Effect size; HbA1c: Glycosylated hemoglobin; HDL: High-density lipoprotein levels; HOMA2-IR: Homeostatic Model Assessment for Insulin resistance; LDL: Low-density lipoprotein levels; RPE: Rate of perceived exertion; SD: Standard deviation; SOT: Sensory organization test; SST: Serum separator tube; T2D: Type 2 diabetes; TUG: Timed up-and-go.
\end{abstract}

\section{Acknowledgements}

The authors would like to acknowledge and thank all the participants for their time and contribution to the present study.

\section{Authors' contributions}

CK, FS and KN contributed to the study conception and design. Material preparation, data collection and analysis were performed by CK, FS and KN. WR, YK and TK helped training sessions and measurements. The first draft of the manuscript was written by CK and all authors contributed to the revisions of the manuscript. All authors read and approved the final manuscript.

\section{Funding}

There was no external funding for the present study.

\section{Availability of data and materials}

The datasets generated and analysed that support the findings of this study are available on reasonable request from the corresponding author [CK]. The data is not publicly available due to them containing information that could compromise research participant privacy/consent.

\section{Declarations}

\section{Ethics approval and consent to participate}

All procedures performed in this study were in accordance with the ethical standards of Edith Cowan University (ECU) and with the 1964 Helsinki declaration and its later amendments. The study was approved by the ECU Human Research Ethics Committee (ID: 19307 KUDIARASU) on November 27th 2017. All participants provided written information consent prior to participation in the study.

\section{Consent for publication}

All participants were informed about the experimental nature of the study and gave their written informed consent for the publication of relevant data.

\section{Competing interests}

The authors declare no conflict of interest with other external parties. The results of the present study are presented in an honest and clear manner.

\section{Author details}

'School of Medical and Health Sciences, Edith Cowan University, Joondalup, WA, Australia. ${ }^{2}$ Exercise Medicine Research Institute, Edith Cowan University, Joondalup, WA, Australia. ${ }^{3}$ Centre for Promotion of Higher Education, Kogakuin University, Tokyo, Japan. ${ }^{4}$ Faculty of Rehabilitation Sciences, Nagoya Gakuin University, Aichi, Japan.
Received: 30 August 2021 Accepted: 6 December 2021

Published online: 20 December 2021

\section{References}

1. Bohannon RW. Reference values for the five-repetition sit-to-stand test: a descriptive meta-analysis of data from elders. Percept Mot Skills. 2006;103(1):215-22

2. Boulé NG. Complex relationship between metformin and exercise in diabetes treatment: should we reconsider our recommendations? Diabetes Manag. 2012;2(1):5-8.

3. Boulé NG, Robert C, Bell GJ, Johnson ST, Bell CR, Lewanczuk RZ, Gabr RQ, Brocks DR. Metformin and exercise in type 2 diabetes: examining treatment modality interactions. Diabetes Care. 2011;34(7):1469-74.

4. Chen TC, Tseng WC, Huang GL, Chen HL, Tseng KW, Nosaka K. Superior effects of eccentric to concentric knee extensor resistance training on physical fitness, insulin sensitivity and lipid profiles of elderly men. Front Physiol. 2017;8:209.

5. Chow BC, Li S, Zhu X, Jiao J, Quach B, Baker JS, Zhang H. Effects of descending or ascending stair exercise on body composition, insulin sensitivity, and inflammatory markers in young Chinese women with obesity: a randomized controlled trial. J Sports Sci. 2021;39(5):496-502.

6. Cohen J. Statistical power analysis for the behavioral sciences. 2nd ed. Hillsdale: Lawrence Erlbaum Associates; 1988.

7. Colberg SR, Albright AL, Blissmer BJ, Braun B, Chasan-Taber L, Fernhall B, Regensteiner JG, Rubin RR, Sigal RJ. Exercise and type 2 diabetes the American college of sports medicine and the American diabetes association: joint position statement. Diabetes Care. 2010;33(12):e147-67.

8. Diabetes Australia. NDSS national diabetes data snapshots. 2019. Retrieved from https://www.ndss.com.au/data-snapshots.

9. Dias CP, Toscan R, de Camargo M, Pereira EP, Griebler N, Baroni BM, Tiggemann CL. Effects of eccentric-focused and conventional resistance training on strength and functional capacity of older adults. Age. 2015;37(5):1-8

10. Drexel $\mathrm{H}$, Mader $\mathrm{A}$, Saely $\mathrm{CH}$, et al. Downhill hiking improves low-grade inflammation, triglycerides, body weight and glucose tolerance. Sci Rep. 2021;11(1):1-8

11. Gault ML, Willems ME. Aging, functional capacity and eccentric exercise training. Aging Dis. 2013;4(6):1-13.

12. Gluchowski A, Harris N, Dulson D, Cronin J. Chronic eccentric exercise and the older adult. Sports Med. 2015;45(10):1413-30.

13. Gordon JP, Thompson BJ, Crane JS, Bressel E, Wagner DR. Effects of isokinetic eccentric versus traditional lower body resistance training on muscle function: examining a multiple-joint short-term training model. Appl Physiol Nutr Metab. 2015;44:118-26.

14. Harada ND, Chiu V, Stewart AL. Mobility-related function in older adults: assessment with a 6-minute walk test. Arch Phys Med Rehabil. 1999;80(7):837-41.

15. Herman T, Giladi N, Hausdorff JM. Properties of the "timed up and go"test: more than meets the eye. Gerontology. 2011;57(3):203-10.

16. Heymsfield SB, Smith R, Aulet M, Bensen B, Lichtman S, Wang J, Piersom RN. Appendicular skeletal muscle mass: measurement by dual-photon absorptiometry. Am J Clin Nutr. 1990;52(2):214-8.

17. Herzog W. Mechanisms of enhanced force production in lengthening (eccentric) muscle contractions. J Appl Physiol. 2014;114:1407-17.

18. Hody S, Croisier JL, Bury T, Rogister B, Leprince P. Eccentric muscle contractions: risks and benefits. Front Physiol. 2019;19:1-18.

19. Hordern MD, Dunstan DW, Prins JB, Baker MK, Singh MAF, Coombes JS. Exercise prescription for patients with type 2 diabetes and pre-diabetes: a position statement from Exercise and Sport Science Australia. J Sci Med Sport. 2012;15(1):25-31.

20. International Diabetes Federation. IDF Diabetes Atlas, 9th edition. 2019. Retrieved from http://www.diabetesatlas.org/.

21. Julian V, Thivel D, Costes F, Touron J, Boirie Y, Pereira B, Perrault H, Duclos $M$, Richard R. Eccentric training improves body composition by inducing mechanical and metabolic adaptations: a promising approach for overweight and obese individuals. Front Physiol. 2018;9:1-14.

22. Julian V, Thivel D, Miguet M, Pereira B, Costes F, Coudeyre E, Duclos M, Richard R. Eccentric cycling is more efficient in reducing fat mass than 
concentric cycling in adolescents with obesity. Scand J Med Sci Sports. 2019;29(1):4-15.

23. Katsura Y, Takeda N, Hara T, Takahashi S, Nosaka K. Comparison between eccentric and concentric resistance exercise training without equipment for changes in muscle strength and functional fitness of older adults. Eur J Appl Physiol. 2019;119(7):1581-90.

24. LaStayo PC, Reich TE, Urquhart M, Hoppeler H, Lindstedt SL. Chronic eccentric exercise: improvements in muscle strength can occur with little demand for oxygen. Am J Physiol. 1999:276(2):R611-5.

25. LaStayo PC, Ewy GA, Pierotti DD, Johns RK, Lindstedt S. The positive effects of negative work: increased muscle strength and decreased fall risk in a frail elderly population. J Gerontol. 2003;58A(5):419-24.

26. LaStayo P, McDonagh P, Lipovic D, Napoles P, Bartholomew A, Esser $K$, Lindstedt $\mathrm{S}$. Elderly patients and high force resistance exercisedescriptive report: can an anabolic, muscle growth response occur without muscle damage or inflammation? J Geriatr Phys Ther. 2007;30:128-34.

27. LaStayo P, Marcus R, Dibble L, Frajacomo F, Lindstedt S. Eccentric exercise in rehabilitation: safety, feasibility, and application. J Appl Physiol. 2014;116(11):1426-34

28. Malin SK, Gerber R, Chipkin SR, Braun B. Independent and combined effects of exercise training and Metformin on insulin sensitivity in individuals with prediabetes. Diabetes Care. 2012;43(12):131-6.

29. Mann RA, Hagy J. Biomechanics of walking, running and sprinting. Am J Sports Med. 1980;8(5):345-50.

30. Marcus RL, Smith S, Morrell G, Addison O, Dibble LE, Wahoff-Stice D, LaStayo PC. Comparison of combined aerobic and high-force eccentric resistance exercise with aerobic exercise only for people with type 2 diabetes mellitus. Phys Ther. 2008;88(11):1345-54.

31. Mueller M, Breil FA, Vogt M, Steiner R, Lippuner K, Popp A, Klossner S, Hoppeler H, Däpp C. Different response to eccentric and concentric training in older men and women. Eur J Appl Physiol. 2009:107(2):145-53.

32. Park SW, Goodpaster BH, Strotmeyer ES, de Rekeneire N, Harris TB, Schwartz AV, Tylaky FA, Newman AB (2006) Decreased muscle strength and quality in older adults with type 2 diabetes: The Health, Aging, and Body Composition Study. Diabetes. 2006;55(6):1813-1818.

33. Paschalis V, Nikolaidis MG, Theodorou AA, Panayiotou G, Fatouros $I G$, Koutedakis $Y$, Jamurtas AZ. A weekly bout of eccentric exercise is sufficient to induce health-promoting effects. Med Sci Sports Exerc. 2011;43(1):64-73.

34. Pesta DH, Goncalves RLS, Madiraju AK, Strasser B, Sparks LM. Resistance training to improve type 2 diabetes: working toward a prescription for the future. Nutr Metab. 2017;14:2-10.

35. Proske U, Morgan DL. Muscle damage from eccentric exercise: mechanism, mechanical signs, adaptation and clinical applications. J Physiol. 2001;537(2):333-45.

36. Pousson M, Lepers R, Hoecke JV. Changes in isokinetic torque and muscular activity of elbow flexors muscles with age. Exp Gerontol. 2001;36(10):1687-98.

37. Raj IS, Bird SR, Westfold BA, Shield A. Effects of eccentrically biased versus conventional weight training in older adults. Med Sci Sports Exerc. 2012:44:1167-76.

38. Reeves ND, Maganaris CN, Longo S, Narici M. Differential adaptations to eccentric versus conventional resistance training in older humans. Exp Physiol. 2009;94(7):825-33.

39. Roig M, O'Brien K, Kirk G, Murray R, McKinnon P, Shadgan B, Reid WD. The effects of eccentric versus concentric resistance training on muscle strength and mass in healthy adults: a systematic review with metaanalysis. Br J Sports Med. 2009;43:556-68.

40. Salkind NJ. Encyclopedia of research design. Thousand Oaks: SAGE; 2010.

41. Sayer AA, Dennison EM, Syddall HE, Gilbody HJ, Phillips DI, Cooper C. Type 2 diabetes, muscle strength, and impaired physical function: the tip of the iceberg? Diabetes Care. 2005;28(10):2541-2.

42. Sharoff CG, Hagobian TA, Malin SK, Chipkin SR, Yu H, Hirshman MF, Goodyear LJ, Braun B. Combining short-term metformin treatment and one bout of exercise does not increase insulin action in insulin-resistant individuals. Am J Physiol Endocrinol Metab. 2010;298:E815-23.

43. Taaffe DR, Duret C, Wheeler S, Marcus R. Once-weekly resistance exercise improves muscle strength and neuromuscular performance in older adults. J Am Geriatr Soc. 1999:47(10):1208-14.

44. Taaffe DR, Cauley JA, Danielson M, Nevitt MC, Lang TF, Bauer DC, et al. Race and sex effects on the association between muscle strength, soft tissue, and bone mineral density in healthy elders: the health, aging, and body composition study. J Bone Miner Res. 2001;16(7):1343-52.

45. Walton RG, Dungan CM, Long DE, Tuggle SC, Kosmac K, Peck BD, Bush HM, Villasante Tezanos AG, McGwin G, Windham ST, Ovalle F, Bamma MM, Kern PA, Peterson CA. Metformin blunts muscle hypertrophy in response to progressive resistance exercise training in older adults: a randomized, double-blind, placebo-controlled, multicenter trial: The MASTERS trial. Aging Cell. 2019;26:1-13.

\section{Publisher's Note}

Springer Nature remains neutral with regard to jurisdictional claims in published maps and institutional affiliations.
Ready to submit your research? Choose BMC and benefit from:

- fast, convenient online submission

- thorough peer review by experienced researchers in your field

- rapid publication on acceptance

- support for research data, including large and complex data types

- gold Open Access which fosters wider collaboration and increased citations

- maximum visibility for your research: over $100 \mathrm{M}$ website views per year

At BMC, research is always in progress.

Learn more biomedcentral.com/submissions 